\title{
High expression of CIP2A protein is associated with tumor aggressiveness in stage I-III NSCLC and correlates with poor prognosis
}

This article was published in the following Dove Press journal:

OncoTargets and Therapy

\author{
Geqi Cha' \\ Jianyu $\mathrm{Xu}$ \\ Xiangying $\mathrm{Xu}$ \\ Bin $\mathrm{Li}^{2}$ \\ Shan Lu' \\ Abiyasi Nanding ${ }^{3}$ \\ Songliu $\mathrm{Hu}^{\prime}$ \\ Shilong Liu' \\ 'Department of Radiation Oncology, \\ Harbin Medical University Cancer \\ Hospital, Harbin, Heilongjiang, \\ ${ }^{2}$ Department of Plastic Surgery, \\ Nanfang Hospital of Southern Medical \\ University, Guangzhou, Guangdong, \\ ${ }^{3}$ Department of Pathology, Harbin \\ Medical University Cancer Hospital, \\ Harbin, Heilongjiang, China
}

\begin{abstract}
The aim of this work was to examine the expression of cancerous inhibitor of protein phosphatase 2A (CIP2A) in non-small cell lung cancer (NSCLC) and analyze its correlation with clinical outcomes. CIP2A protein levels were detected by immunohistochemistry (IHC). One hundred and eighty-four of 209 (88.3\%) primary stage I-III NSCLC specimens and 4 of $38(10.5 \%)$ adjacent normal lung tissue specimens expressed CIP2A protein. High expression of CIP2A was detected in $38.8 \%(81 / 209)$ of the NSCLC specimens. Patients diagnosed histologically with late-stage NSCLC $(p<0.001)$ and malignant nodes $(p=0.001)$ exhibited high CIP2A expression. Univariate analysis using the log-rank test identified CIP2A expression as a prognostic predictor for overall survival $(p=0.005)$. In multivariate analyses using the Cox regression test, CIP2A expression, T stage, $\mathrm{N}$ stage, histological type, and chemotherapy were identified as independent prognostic factors $(p=0.007,0.001,0.003,<0.001$, and $<0.001$, respectively). Furthermore, Kaplan-Meier survival curves demonstrated that high CIP2A expression indicated poor prognosis in the subgroup of patients with squamous cell carcinoma ( $p=0.008)$. Similar results were noted in the subgroup of patients with adenocarcinoma, but the results did not reach statistical significance $(p=0.084)$. We also used univariate analysis and multivariate analysis to assess the prognostic factors for overall survival in the subgroup of patients who received postoperative chemotherapy. CIP2A expression was also an independent prognostic factor in NSCLC patients who received postoperative chemotherapy ( $p=0.009)$, along with histological type $(p=0.001)$ and $\mathrm{N}$ stage $(p=0.034)$. In conclusion, adding to the accumulating evidence, our research suggested that the CIP2A expression is associated with aggressiveness and correlates with poor prognosis in NSCLC. Our findings also indicated that CIP2A might be a potential therapeutic target against NSCLC.
\end{abstract}

Keywords: CIP2A, non-small cell lung carcinoma, immunohistochemistry, chemotherapy, radiotherapy, prognosis

\section{Introduction}

Lung cancer is the leading cause of cancer death worldwide, ${ }^{1}$ and $80 \%$ of cases involve non-small cell lung cancer (NSCLC). Despite the numerous different treatment options available, including surgery, chemotherapy, radiation therapy, and targeted therapy, lung cancer cure rates have increased slowly over recent decades. As an indicator of cure rates, median 5-year survival rate ranges from $\sim 50 \%$ for stage IA disease to $<30 \%$ for stage IIIA NSCLC. ${ }^{2}$ Novel predictive biomarkers and therapeutic targets are required to improve survival rate.

As a human oncoprotein, cancerous inhibitor of protein phosphatase 2A (CIP2A) was recently identified as a PP2A (protein phosphatase 2A) inhibitor and MYC
Correspondence: Xiangying Xu Department of Radiation Oncology, Harbin Medical University Cancer Hospital, I50 Haping Road, Nangang District, Harbin I5008I, China $\mathrm{Tel} / \mathrm{fax}+8645186298521$

Email xxyyxu666@163.com 
(Transcription factor p64) stabilizer in human malignancies. ${ }^{3}$ Furthermore, there is a positive-feedback loop between CIP2A and MYC that may account for the pan expression and activation of MYC protein in cancer cells. ${ }^{4,5}$ In addition, CIP2A promotes the phosphorylation and activity of other oncoproteins, such as E2F1 (E2F transcription factor 1) and AKT (protein kinase B). ${ }^{6,7}$ Various independent studies have validated CIP2A's role in promoting tumor growth and resistance to apoptosis and senescence-inducing therapies. In clinical studies, CIP2A overexpression has been confirmed in solid and hematological cancers. ${ }^{3,4,8-10}$ Moreover, CIP2A overexpression predicts poor prognosis in several human cancers, such as gastric cancer, ovarian cancer, and tongue cancer. ${ }^{4,8,10}$

By examining CIP2A expression in human NSCLC specimens using immunohistochemistry (IHC), this study aimed not only to investigate its relationship with clinicopathological variables and prognostic factors but also sought to assess its correlation with prognosis in different histological types. We also explored the influence of CIP2A expression on postoperative chemotherapeutic or radiotherapeutic clinical treatments.

\section{Materials and methods}

\section{Patients and tissue specimens}

This retrospective study was approved by the Medical Ethical Committee of Harbin Medical University Cancer Hospital, Harbin, China. All patients provided written informed consent for participation in this study. Two hundred and nine primary NSCLC specimens and 38 paired adjacent normal lung tissue specimens were used in this research. The specimens were obtained from patients diagnosed with stage IA-IIIB NSCLC who underwent surgeries at the Harbin Medical University Cancer Hospital between March 2005 and December 2007. These patients did not receive any therapy before operation. The median age of the cohort was 58 years. The TNM staging system for lung cancer was used to classify patients as stages I $(n=59)$, II $(n=81)$, and III $(n=69) .{ }^{11}$ In pathological diagnosis, 116 tumors were squamous cell carcinomas and 93 were adenocarcinomas. A total of 151 patients received three to four cycles of adjuvant platinum doublet chemotherapy, and 58 patients received postoperative radiotherapy at a total dose that ranged from 50 to 66 Gy with 2 Gy per fraction administered 5 days per week. Complete clinical and follow-up data were available for all patients.

\section{Immunohistochemistry}

Immunohistochemical staining was performed according to the manufacturer's protocol of the PV-6001 kit (Zhong Shan
Golden Bridge Biological Technology Inc., Beijing, China). Briefly, 4- $\mu \mathrm{m}$ sections were deparaffinized with xylene, rehydrated in a decreasing ethanol gradient, and rinsed in phosphate-buffered saline. The slides were immersed in hydrogen peroxide (3\%) to block endogenous peroxide activity for $15 \mathrm{~min}$ at room temperature. Antigen retrieval was performed by placing the slides in citrate buffer $(0.01 \mathrm{M}$, $\mathrm{pH}$ 6.0) heated in a pressure cooker for $8 \mathrm{~min}$. Then, the specimens were incubated with the CIP2A primary antibody (1:50, rabbit polyclonal; ab84547; Abcam, Cambridge, UK) at $4^{\circ} \mathrm{C}$ overnight. After incubation with biotin-labeled secondary antibodies at room temperature for $30 \mathrm{~min}$, positive staining was detected by diaminobenzidine (DAB) reagent sets. Then, the specimens were counterstained with hematoxylin. Finally, each section was dehydrated by gradient alcohol and covered with a coverslip.

\section{Immunohistochemical staining evaluation}

All slides were examined by two experienced pathologists in a double-blinded fashion. The CIP2A expression level was classified by the product of intensity and proportion of positively stained cells. Positive staining presented as brownyellow particles. The staining intensity was classified based on the following criteria: 0 (no staining), 1 (light yellow/weak staining), 2 (yellow-brown/moderate staining), or 3 (brown/ strong staining). The proportion of positively stained tumor cells was scored as follows: 1 (positive cells 1\%-25\%), 2 (positive cells 26\%-50\%), 3 (positive cells 51\%-75\%), or 4 (positive cells $75 \%-100 \%$ ). ${ }^{12}$ The scores of each tumor sample were multiplied to yield a final score of $0-12$, and the median value of score 3 was used to distinguish low versus high CIP2A expression. The specimen with a final score of $\leq 3$ was classified as having low expression. Otherwise, the specimen was classified as having high expression.

\section{Statistical analysis}

Statistical analysis was performed using the SPSS 21.0 software (IBM Corp., Armonk, NY, USA). Categorical variables were analyzed by Pearson's chi-square test or Fisher's exact test. Survival analyses were calculated by the Kaplan-Meier method with log-rank test and Cox regression method. The $p$-values $<0.05$ were considered statistically significant.

\section{Results \\ CIP2A expression profile in NSCLC}

CIP2A protein expression in NSCLC samples and adjacent normal tissues was analyzed by IHC. Positive CIP2A staining was predominantly localized within the cytoplasm and detected in $184(88.3 \%)$ out of the 209 tumors and 4 (10.5\%) 

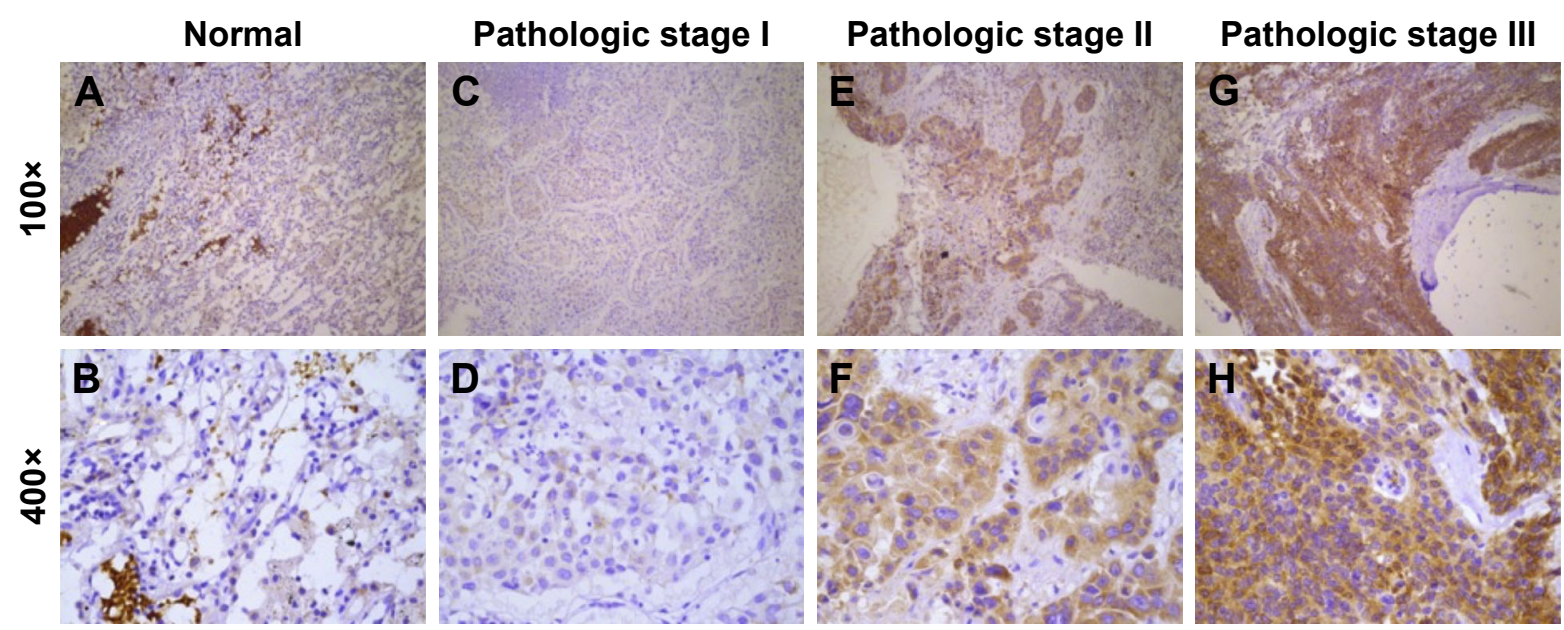

Figure I Immunohistochemical staining of CIP2A in NSCLC specimens.

Notes: Positive CIP2A protein staining was mainly observed in cytoplasm. (A and B) CIP2A expression in adjacent normal lung tissue. The final score is 0 (negative). (C and D) CIP2A expression in primary NSCLC tissues of pathologic stage I. The final score is I (IXI). (E and F) CIP2A expression in primary NSCLC tissues of pathologic stage II. The final score is $4(2 \times 2)$. ( $(\mathbf{G}$ and $\mathbf{H})$ CIP2A expression in primary NSCLC tissues of pathologic stage III. The final score is 12 (4×3)

Abbreviations: CIP2A, cancerous inhibitor of protein phosphatase 2A; NSCLC, non-small cell lung cancer.

out of 38 adjacent normal lung tissues. Based on IHC scoring, $128(61.2 \%)$ tumor specimens exhibited low expression and $81(38.8 \%)$ tumor specimens exhibited high CIP2A expression and all the adjacent normal lung tissue specimens were characterized with CIP2A low expression. Overall, CIP2A was highly detectable in tumor cells compared with adjacent normal lung tissues (Figures 1 and 2).

\section{Correlation between CIP2A expression and clinicopathological variables}

Next, we assessed whether CIP2A expression correlated with clinicopathological parameters in NSCLC. Table 1 demonstrates that CIP2A expression was significantly associated with high pathologic stage and N status. Patients diagnosed with a higher pathologic stage $(p<0.001)$ and $\mathrm{N}$ stage ( $p=0.001$ ) exhibited increased CIP2A expression. However, no significant differences were observed regarding diagnosis, age, gender, smoking, histological type, differentiation grade, and tumor status (all $p>0.05$ ).

\section{Clinical impact of CIP2A expression on survival}

In survival analyses, the median time of overall survival (OS) was 50.0 months (95\% CI, 35.83-64.17 months).
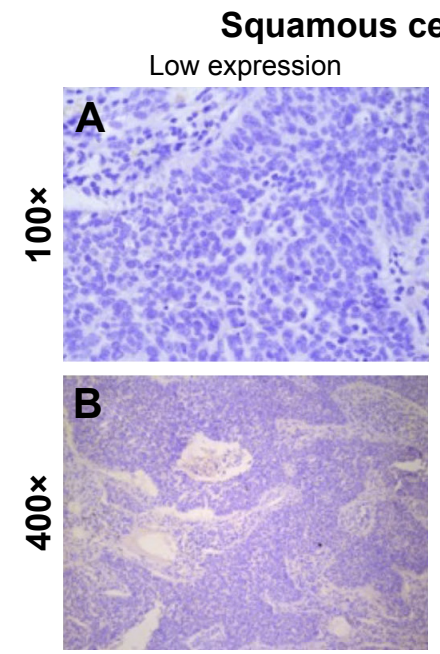
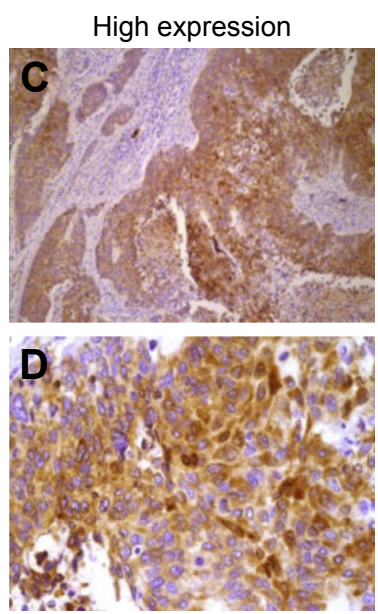

\section{Adenocarcinoma}

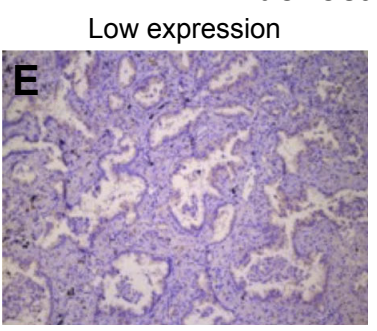

High expression
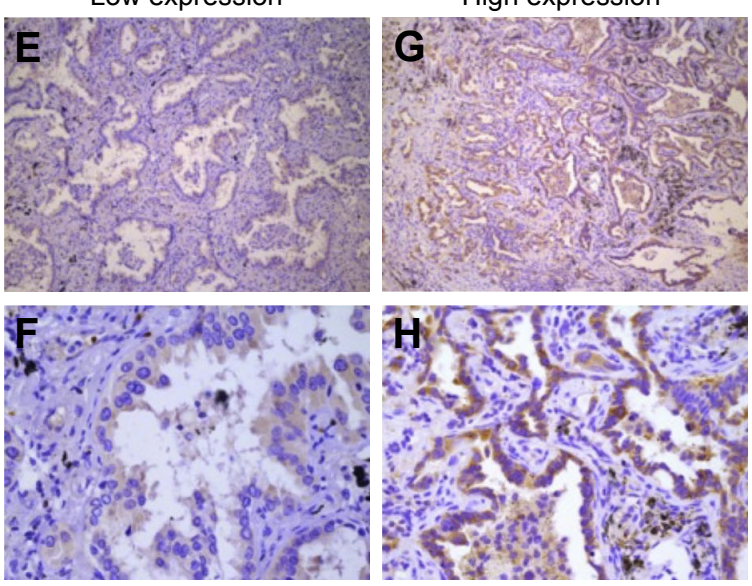

Figure 2 Immunohistochemical staining of CIP2A in squamous cell carcinoma and adenocarcinoma.

Notes: (A and B) Low expression of CIP2A in squamous cell carcinoma. (C and D) High expression of CIP2A in squamous cell carcinoma. (E and F) Low expression of CIP2A in adenocarcinoma. ( $\mathbf{G}$ and $\mathbf{H}$ ) High expression of CIP2A in adenocarcinoma.

Abbreviation: CIP2A, cancerous inhibitor of protein phosphatase $2 \mathrm{~A}$. 
Table I Clinicopathological variables of patient samples and expression of CIP2A in NSCLC

\begin{tabular}{|c|c|c|c|c|c|}
\hline Variables & $\begin{array}{l}\text { Patients } \\
\text { (n) }\end{array}$ & $\begin{array}{l}\text { CIP2A } \\
\text { low (\%) }\end{array}$ & $\begin{array}{l}\text { CIP2A } \\
\text { high (\%) }\end{array}$ & $\chi^{2}$ & $p$-value \\
\hline Age (years) & & & & 1.006 & 0.361 \\
\hline$\leq 58$ & 107 & $62(57.9)$ & $45(42.1)$ & & \\
\hline$>58$ & 102 & $66(64.7)$ & $36(35.3)$ & & \\
\hline Gender & & & & 0.031 & 0.860 \\
\hline Male & 156 & 95 (60.9) & 61 (39.I) & & \\
\hline Female & 53 & $33(62.3)$ & $20(37.7)$ & & \\
\hline Smoking history & & & & 0.864 & 0.353 \\
\hline Yes & 132 & $84(63.6)$ & $48(36.4)$ & & \\
\hline No & 77 & $44(57.1)$ & $33(42.9)$ & & \\
\hline Pathologic stage & & & & 15.642 & $0.000 *$ \\
\hline 1 & 59 & $48(81.4)$ & II (I8.6) & & \\
\hline ॥ & 81 & $47(58.0)$ & $34(42.0)$ & & \\
\hline III & 69 & $33(47.8)$ & $36(52.2)$ & & \\
\hline T stage & & & & 1.578 & 0.454 \\
\hline $\mathrm{TI}$ & 39 & $23(59.0)$ & $16(41.0)$ & & \\
\hline $\mathrm{T} 2$ & 148 & $94(63.5)$ & $54(36.5)$ & & \\
\hline $\mathrm{T} 3+\mathrm{T} 4$ & 22 & II (50.0) & II (50.0) & & \\
\hline $\mathrm{N}$ stage & & & & 14.332 & $0.001 *$ \\
\hline No & 104 & $77(74.0)$ & $27(26.0)$ & & \\
\hline $\mathrm{NI}$ & 40 & $20(58.0)$ & $20(42.0)$ & & \\
\hline N2 & 65 & $31(47.8)$ & $34(52.2)$ & & \\
\hline Histological type & & & & 1.334 & 0.248 \\
\hline $\begin{array}{l}\text { Squamous cell } \\
\text { carcinoma }\end{array}$ & 116 & $67(57.8)$ & $49(42.2)$ & & \\
\hline Adenocarcinoma & 93 & $61(65.6)$ & $32(34.4)$ & & \\
\hline Differentiation grade & & & & 4.914 & 0.086 \\
\hline Well & 27 & $14(5 \mid .9)$ & I3 (48.I) & & \\
\hline Moderate & 90 & $50(55.6)$ & $40(44.4)$ & & \\
\hline Poor & 92 & $64(69.6)$ & $28(30.4)$ & & \\
\hline
\end{tabular}

Note: *Significant difference.

Abbreviations: CIP2A, cancerous inhibitor of protein phosphatase 2A; NSCLC, non-small cell lung cancer.

The univariate analysis identified six variables that exhibit statistically significant associations with OS in all patients: CIP2A expression $(p=0.005)$, pathologic stage $(p=0.005)$, $\mathrm{N}$ stage $(p=0.001)$, histological type $(p=0.003)$, postoperative chemotherapy $(p=0.037)$, and postoperative radiotherapy ( $p=0.006$ ) (Table 2). Kaplan-Meier survival curves demonstrated that high CIP2A expression was a predictor of poor prognosis in NSCLC patients $(p=0.005$, Figure $3 \mathrm{~A}$ ). The median OS of patients with high CIP2A expression and low CIP2A expression were 31.0 months (95\% CI, 23.79-38.21 months) and 68.0 months (95\% CI, 48.20-87.80 months), respectively.

In addition, multivariate Cox proportional hazard analysis demonstrated that high CIP2A expression $(p=0.007)$ was an independent prognostic factor along with $\mathrm{T}$ stage $(p=0.001), \mathrm{N}$ stage $(p=0.003)$, histological type $(p<0.001)$, and chemotherapy $(p<0.001)$ (Table 2). Overall, high CIP2A expression is a poor and independent prognostic factor for postoperative stage I-III NSCLC patients.

\section{Clinical impact of CIP2A expression on survival in subgroups}

Furthermore, we analyzed the relationship between OS and CIP2A expression in different subgroups. For patients diagnosed with squamous cell carcinoma $(\mathrm{n}=116$; 49 high CIP2A expression and 67 low CIP2A expression), Kaplan-Meier survival curves demonstrated that high CIP2A expression indicated poor prognosis in NSCLC patients with squamous cell carcinoma ( $p=0.008$, Figure $3 \mathrm{~B}$ ). A trend toward worse OS was noted in patients with high CIP2A expression and adenocarcinoma ( $\mathrm{n}=93 ; 32$ high CIP2A expression and 61 low CIP2A expression), but the trend did not achieve statistical significance $(p=0.084$, Figure $3 \mathrm{C})$.

The median OS of patients who received postoperative chemotherapy with high $(\mathrm{n}=67)$ and low $(\mathrm{n}=84)$ expressions of CIP2A were 35.0 months (95\% CI, 21.97-48.03 months) and 80.0 months (95\% CI, 54.85-105.15 months), respectively ( $p=0.005$, Figure $3 \mathrm{D}$ ). Multivariate analysis revealed that CIP2A expression $(p=0.009)$, histological type $(p=0.001)$, and $\mathrm{N}$ stage $(p=0.034)$ were independent prognostic factors for OS in patients who received postoperative chemotherapy (Table 3). Overall, high CIP2A expression was a poor and independent prognostic factor for stage I-III NSCLC patients who received postoperative chemotherapy. In the subgroup of patients who received postoperative radiotherapy, a log-rank test demonstrated that high CIP2A expression correlated with poor OS $(p=0.165)$. The median OS values of patients who received postoperative radiotherapy with high $(n=31)$ and low $(n=27)$ CIP2A expressions were 21.0 months (95\% CI, 10.87-31.13 months) and 36.0 months (95\% CI, 24.13-47.87 months), respectively. However, the results were not statistically significant.

\section{Discussion}

In this study, we demonstrated that CIP2A protein expression in lung cancer tissues was dramatically increased compared with normal lung tissues. As determined by IHC, CIP2A expression was detected in 184 (88.3\%) stage I-III NSCLC specimens and in $4(10.5 \%)$ adjacent normal lung tissues. Junttila et al demonstrated that CIP2A mRNA levels were very low in most nonmalignant samples, except testis, bone marrow, brain, cerebellum, and prostate. ${ }^{3}$ They also confirmed high CIP2A protein expression in head and neck squamous cell carcinoma (HNSCC) and negative staining of CIP2A in all nine nonmalignant tissues. This finding indicated that CIP2A expression was 
Table 2 Univariate and multivariate analyses of the prognostic factors in 209 patients with NSCLC

\begin{tabular}{|c|c|c|c|c|c|}
\hline \multirow[t]{2}{*}{ Variables } & \multirow{2}{*}{$\begin{array}{l}\text { Patients } \\
\text { (n) }\end{array}$} & \multicolumn{2}{|l|}{ Univariate } & \multicolumn{2}{|l|}{ Multivariate } \\
\hline & & $\begin{array}{l}\text { Median survival time } \\
\text { (months) }(95 \% \mathrm{Cl})\end{array}$ & p-value ${ }^{a}$ & HR $(95 \% \mathrm{Cl})$ & $p$-value ${ }^{b}$ \\
\hline Age (years) & & & 0.234 & & \\
\hline$\leq 58$ & 107 & $50.0(23.3-76.7)$ & & & \\
\hline$>58$ & 102 & $50.0(34.8-65.2)$ & & & \\
\hline Gender & & & 0.574 & & \\
\hline Male & 156 & $44.0(24.9-63.1)$ & & & \\
\hline Female & 53 & $54.0(43.3-64.7)$ & & & \\
\hline Smoking history & & & 0.337 & & \\
\hline Yes & 132 & $39.0(23.2-54.8)$ & & & \\
\hline No & 77 & $59.0(46.6-71.4)$ & & & \\
\hline Pathologic stage & & & $0.005^{*}$ & & \\
\hline I & 59 & $75.0(53.5-96.5)$ & & & \\
\hline ॥ & 81 & $59.0(34.5-83.5)$ & & & \\
\hline III & 69 & $31.0(25.9-36.1)$ & & & \\
\hline$N$ stage & & & $0.001 *$ & 1.441 (I.I34-I.830) & $0.003 *$ \\
\hline No & 104 & $75.0(56.6-93.4)$ & & & \\
\hline NI & 40 & $44.0(13.0-75.0)$ & & & \\
\hline N2 & 65 & $30.0(25.1-34.9)$ & & & \\
\hline T stage & & & 0.068 & $1.749(1.264-2.421)$ & $0.001 *$ \\
\hline $\mathrm{TI}$ & 39 & $68.0(-)^{c}$ & & & \\
\hline $\mathrm{T} 2$ & 148 & $49.0(31.8-66.2)$ & & & \\
\hline $\mathrm{T} 3+\mathrm{T} 4$ & 22 & $21.0(6.1-35.9)$ & & & \\
\hline Histological type & & & $0.003 *$ & $1.949(1.384-2.746)$ & $<0.00 I^{*}$ \\
\hline Squamous cell carcinoma & 116 & $61.0(32.0-90.0)$ & & & \\
\hline Adenocarcinoma & 63 & $39.0(24.0-54.0)$ & & & \\
\hline Differentiation grade & & & 0.975 & & \\
\hline Well & 27 & $57.0(27.7-86.3)$ & & & \\
\hline Moderate & 90 & $49.0(30.4-67.6)$ & & & \\
\hline Poor & 92 & $50.0(26.5-73.5)$ & & & \\
\hline CIP2A expression & & & $0.005^{*}$ & $1.616(1.137-2.298)$ & $0.005^{*}$ \\
\hline Low & 128 & $68.0(48.2-87.8)$ & & & \\
\hline High & 81 & $31.0(23.8-38.2)$ & & & \\
\hline Chemotherapy & & & $0.037^{*}$ & 2.543 (1.703-3.799) & $0.000^{*}$ \\
\hline Yes & $|5|$ & $57.0(37.9-76.1)$ & & & \\
\hline No & 58 & $24.0(\mid 6.5-31.5)$ & & & \\
\hline Radiotherapy & & & $0.006 *$ & $\mathrm{I} .304(0.845-2.0 \mathrm{II})$ & 0.230 \\
\hline Yes & 58 & $30.0(22.5-37.5)$ & & & \\
\hline No & 151 & $64.0(49.1-78.9)$ & & & \\
\hline
\end{tabular}

Notes: a Log-rank test. ${ }^{\circ}$ Cox regression test. 'Because of the relative high percentage (46.2\%) of censored data in TI group, the SPSS software didn't show the $95 \%$ Cl. *Significant difference.

Abbreviations: $\mathrm{Cl}$, confidence interval; CIP2A, cancerous inhibitor of protein phosphatase 2A; HR, hazard ratio; NSCLC, non-small cell lung cancer.

significantly increased in tumor tissues compared with adjacent normal tissues, which is consistent with other studies. ${ }^{3,13}$

Given that CIP2A was identified as a new oncoprotein that stabilizes the function of c-MYC and is overexpressed in HNSCC and colon cancer, ${ }^{3} \mathrm{CIP} 2 \mathrm{~A}$ overexpression has been detected in a variety of tumor types. ${ }^{3,8,14-16}$ In NSCLC, Ma et al reported that CIP2A is detected in $63.3 \%$ (by Western blotting) and $67.2 \%$ (at the mRNA level) of tumor specimens. ${ }^{17}$ Dong et al reported that CIP2A is expressed in $82.7 \%$ of cases at the mRNA level and in $72.2 \%$ of cases at the protein level (detected by IHC). ${ }^{12}$ In our research, CIP2A protein levels were detected in a higher proportion $(88.3 \%)$ of the cohort compared with previous studies. Liu et al reported that CIP2A protein was detected in $90.3 \%$ of nasopharyngeal cancer samples analyzed. ${ }^{16}$ In the review by Khanna et $\mathrm{al}^{14}$ several presumed oncogenic signaling mechanisms that drive the expression of CIP2A were summarized, including MYC, the EGFR-MEK-ETS1 pathway, p53 inactivation, and E2F1 and ATF-2 (activating transcription factor 2) overexpression. Khanna recently identified CHK1 (checkpoint kinase 1), a DNA-damage kinase, as a stimulator of CIP2A transcription in cancer cells. CHK1 is constitutively phosphorylated by another DNA-damage kinase DNA-PK. ${ }^{18}$ The above results may explain the widespread 

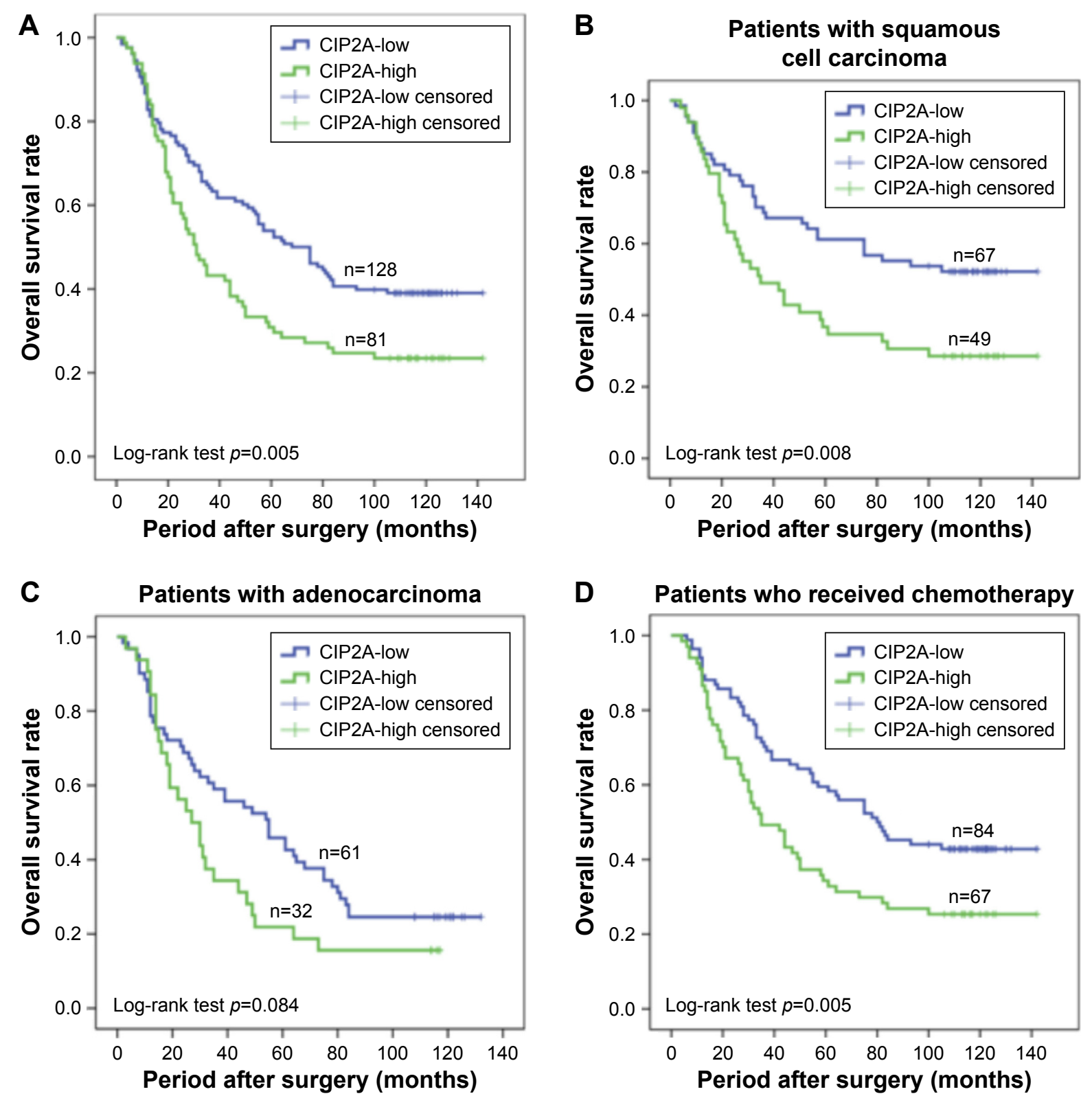

Figure 3 Kaplan-Meier curves of OS based on CIP2A expression in NSCLC patients.

Notes: (A) OS analysis of all patients. (B) OS analysis of squamous cell carcinoma patients. (C) OS analysis of adenocarcinoma patients. (D) OS analysis of patients who received postoperative chemotherapy.

Abbreviations: CIP2A, cancerous inhibitor of protein phosphatase 2A; NSCLC, non-small cell lung cancer; OS, overall survival.

Table 3 Multivariate analysis of the prognostic factors using Cox regression test in I5I NSCLC patients who received postoperative chemotherapy

\begin{tabular}{llll}
\hline Variable & HR & $\mathbf{9 5 \%} \mathbf{C l}$ & p-value \\
\hline T stage & 1.387 & $0.945-2.036$ & 0.095 \\
$\mathrm{~N}$ stage & 1.336 & $1.023-1.747$ & $0.034^{*}$ \\
Histological type & 2.039 & $1.353-3.072$ & $0.00 I^{*}$ \\
CIP2A expression & 1.720 & $1.148-2.577$ & $0.009^{*}$ \\
Radiotherapy & 1.356 & $0.844-2.18 \mathrm{I}$ & 0.208 \\
\hline
\end{tabular}

Note: *Significant difference.

Abbreviations: $\mathrm{Cl}$, confidence interval; $\mathrm{CIP} 2 \mathrm{~A}$, cancerous inhibitor of protein phosphatase 2A; HR, hazard ratio; NSCLC, non-small cell lung cancer. overexpression of CIP2A across multiple human cancer types due to the numerous factors driving CIP2A expression.

Furthermore, we found that high CIP2A expression is associated with late pathologic stage and metastatic lymph node status in NSCLC. Several studies also demonstrated similar phenomena in breast cancer, ${ }^{15,19}$ gastric cancer, tongue cancer, ovarian cancer, and renal cell cancer., ${ }^{4,8,10,20}$ In our study, the correlation between CIP2A protein expression and pathologic stage $(p<0.001)$ in NSCLC is consistent with $\mathrm{Xu}$ et al's research. ${ }^{21}$ We demonstrated for the first time that 
CIP2A overexpression is associated with lymph node status in NSCLC, further supporting a role for CIP2A in promoting cancer aggressiveness. The possible explanations for the discrepancies in the outcomes between our research and the other studies that failed to identify the association between CIP2A expression and tumor aggressiveness in NSCLC could be the difference in sample size and the use of the median score as the cutoff point. Alternatively, the use of different antibodies for CIP2A sample staining could further explain the disparity. However, the mechanism of CIP2A in tumor migration is unclear. Ren et al reported that CIP2A depletion by siRNA resulted in both inhibition of c-MYC protein expression and migration in renal cells. ${ }^{20}$ Based on the role of CIP2A in stabilizing and upregulating the MYC oncoprotein, it is likely that MYC is involved in CIP2A-stimulated tumor cell invasion. Sung et al demonstrated that IL-10-mediated tumor aggressiveness in vivo and in vitro occurs through increased CIP2A expression via the PI3K signaling pathway. ${ }^{22}$

In addition, we confirmed that high CIP2A expression predicted poor prognosis in stage I-III NSCLC. CIP2A overexpression has been established as a prognostic biomarker in various cancers, including gastric cancer, bladder cancer, ovarian cancer, tongue cancer, colon cancer, chronic myelogenous leukemia, and NSCLC. ${ }^{4,8-10,12,23}$ Dong et al found that patients diagnosed with CIP2A-positive NSCLC exhibited reduced survival compared with patients diagnosed with CIP2A-negative NSCLC. ${ }^{12}$ This result is consistent with $\mathrm{Xu}$ et al's and our study. ${ }^{21}$

We also performed survival analyses on CIP2A expression in patients with different histological types and found that high CIP2A expression is a prognosis factor in squamous cell carcinoma ( $p=0.008$, Figure $3 \mathrm{~B}$ ). High expression of CIP2A might be a prognostic factor even in adenocarcinoma; however, the result did not achieve statistical significance ( $p=0.084$, Figure 3 C). High CIP2A expression indicated poor prognosis in other squamous cell cancers, such as tongue cancer and nasopharyngeal cancer. ${ }^{10,16}$ However, a study of colorectal cancer by Bockelman et al demonstrated that CIP2A expression was not associated with patient prognosis. ${ }^{24}$ Khanna et al demonstrated that the prognostic role of CIP2A was established only in particular subgroups of gastric cancer patients. ${ }^{4}$ Of course, the change in sample size might account for the discrepancies between all patients and subgroups of adenocarcinoma patients. Differences in the role of CIP2A carcinogenesis in squamous cell cancer and adenocarcinoma should be validated by further investigations.

CIP2A is defined as an oncoprotein, based on its relatively low expression in normal lung tissues and its correlation with oncogenic pathways, and it serves as a promising molecular target for cancer therapy. In multivariate survival analysis, CIP2A overexpression predicts poor outcomes in the cohort undergoing postoperative chemotherapy. Previous studies indicate that high CIP2A expression is related to chemotherapy resistance. ${ }^{6,25}$ Laine et al demonstrated that CIP2A confers resistance to vinorelbine in HER2-negative breast cancers. ${ }^{6}$ Moreover, Wei et al reported that CIP2A overexpression reduced NSCLC cell chemosensitivity to cisplatin by activating the Akt pathway. ${ }^{26}$ Vinorelbine and platinum were both used broadly in NSCLC chemotherapy. Based on all the aforementioned results, a combination of chemotherapy and targeted therapy against CIP2A in NSCLC may be more effective. A recent study identified CIP2A as a target gene of Oct4 (a stem cell transcription factor) and provided evidence that these genes cooperate in radioresistance in HNSCC. ${ }^{27} \mathrm{We}$ also analyzed CIP2A protein expression and the prognosis in the subgroup of NSCLC patients who received postoperative radiotherapy, but we did not identify a correlation between CIP2A expression and OS. The limited number of patients who received postoperative radiotherapy in our study $(n=58)$ might explain this finding. Additional data and further research are required to make a final conclusion.

\section{Conclusion}

In summary, our research demonstrates that high CIP2A expression is associated with cancer aggressiveness and poor prognosis in NSCLC, especially in squamous cell carcinoma and in patients who received postoperative chemotherapy. Our results indicated that CIP2A might be a promising predictive biomarker and therapeutic target in NSCLC. Further studies are required to understand the underlying molecular and cellular mechanisms of CIP2A in NSCLC.

\section{Acknowledgments}

We thank all the patients and their families who participated in this study. We are also grateful to Xia Bingshu and Zhang Siliang for their technical help and fruitful discussions.

\section{Disclosure}

The authors report no conflicts of interest in this work.

\section{References}

1. Torre LA, Bray F, Siegel RL, Ferlay J, Lortet-Tieulent J, Jemal A. Global cancer statistics, 2012. CA Cancer J Clin. 2015;65(2):87-108.

2. Groome PA, Bolejack V, Crowley JJ, et al. The IASLC lung cancer staging project: validation of the proposals for revision of the T, N, and $\mathrm{M}$ descriptors and consequent stage groupings in the forthcoming (seventh) edition of the TNM classification of malignant tumours. J Thorac Oncol. 2007;2(8):694-705 
3. Junttila MR, Puustinen P, Niemelä M, et al. CIP2A Inhibits PP2A in human malignancies. Cell. 2007;130(1):51-62.

4. Khanna A, Böckelman C, Hemmes A, et al. MYC-dependent regulation and prognostic role of CIP2A in gastric cancer. J Natl Cancer Inst. 2009;101(11):793-805.

5. Mathiasen DP, Egebjerg C, Andersen SH, et al. Identification of a c-Jun N-terminal kinase-2-dependent signal amplification cascade that regulates c-Myc levels in ras transformation. Oncogene. 2011;31(3): 390-401.

6. Laine A, Sihto H, Come C, et al. Senescence sensitivity of breast cancer cells is defined by positive feedback loop between CIP2A and E2F1. Cancer Discov. 2013;3(2):182-197.

7. Chen K-F, Liu C-Y, Lin Y-C, et al. CIP2A mediates effects of bortezomib on phospho-Akt and apoptosis in hepatocellular carcinoma cells. Oncogene. 2010;29(47):6257-6266.

8. Bockelman C, Lassus H, Hemmes A, et al. Prognostic role of CIP2A expression in serous ovarian cancer. Br J Cancer. 2011;105(7): 989-995.

9. Lucas CM, Harris RJ, Giannoudis A, Copland M, Slupsky JR, Clark RE. Cancerous inhibitor of PP2A (CIP2A) at diagnosis of chronic myeloid leukemia is a critical determinant of disease progression. Blood. 2011; 117(24):6660-6668.

10. Bockelman C, Hagstrom J, Makinen LK, et al. High CIP2A immunoreactivity is an independent prognostic indicator in early-stage tongue cancer. Br J Cancer. 2011;104(12):1890-1895.

11. Detterbeck FC, Boffa DJ, Tanoue LT. The new lung cancer staging system. Chest. 2009;136(1):260-271.

12. Dong Q-Z, Wang Y, Dong X-J, et al. CIP2A is overexpressed in nonsmall cell lung cancer and correlates with poor prognosis. Ann Surg Oncol. 2011;18(3):857-865.

13. $\mathrm{Li} \mathrm{W}, \mathrm{Ge} \mathrm{Z}$, Liu C, et al. CIP2A is overexpressed in gastric cancer and its depletion leads to impaired clonogenicity, senescence, or differentiation of tumor cells. Clin Cancer Res. 2008;14(12):3722-3728.

14. Khanna A, Pimanda JE, Westermarck J. Cancerous inhibitor of protein phosphatase $2 \mathrm{~A}$, an emerging human oncoprotein and a potential cancer therapy target. Cancer Res. 2013;73(22):6548-6553.

15. Come C, Laine A, Chanrion M, et al. CIP2A is associated with human breast cancer aggressivity. Clin Cancer Res. 2009;15(16): 5092-5100.
16. Liu N, He Q-M, Chen J-W, et al. Overexpression of CIP2A is an independent prognostic indicator in nasopharyngeal carcinoma and its depletion suppresses cell proliferation and tumor growth. Mol Cancer. 2014;13(1):1-9.

17. Ma L, Wen Z-S, Liu Z, et al. Overexpression and small moleculetriggered downregulation of CIP2A in lung cancer. PLoS One. 2011; 6(5):e20159.

18. Khanna A, Kauko O, Bockelman C, et al. Chk1 targeting reactivates PP2A tumor suppressor activity in cancer cells. Cancer Res. 2013; 73(22):6757-6769

19. Yu G, Liu G, Dong J, Jin Y. Clinical implications of CIP2A protein expression in breast cancer. Med Oncol. 2013;30(2):524.

20. Ren J, Li W, Yan L, et al. Expression of CIP2A in renal cell carcinomas correlates with tumour invasion, metastasis and patients' survival. Br J Cancer. 2011;105(12):1905-1911.

21. Xu P, Xu X-L, Huang Q, Zhang Z-H, Zhang Y-B. CIP2A with survivin protein expressions in human non-small-cell lung cancer correlates with prognosis. Med Oncol. 2011;29(3):1643-1647.

22. Sung W-W, Wang Y-C, Lin P-L, et al. IL-10 promotes tumor aggressiveness via upregulation of CIP2A transcription in lung adenocarcinoma. Clin Cancer Res. 2013;19(15):4092-4103.

23. Xue $\mathrm{Y}, \mathrm{Wu} \mathrm{G}$, Wang $\mathrm{X}$, et al. CIP2A is a predictor of survival and a novel therapeutic target in bladder urothelial cell carcinoma. Med Oncol. 2012;30(1):10-11.

24. Böckelman C, Koskensalo S, Hagström J, Lundin M, Ristimäki A, Haglund C. CIP2A overexpression is associated with c-Myc expression in colorectal cancer. Cancer Biol Ther. 2012;13(5):289-295.

25. Choi YA, Park JS, Park MY, et al. Increase in CIP2A expression is associated with doxorubicin resistance. FEBS Lett. 2011;585(5):755-760.

26. Wei L, Qu W, Sun J, et al. Knockdown of cancerous inhibitor of protein phosphatase 2A may sensitize NSCLC cells to cisplatin. Cancer Gene Ther. 2014;21(5):194-199.

27. Ventelä S, Sittig E, Mannermaa L, et al. CIP2A is an Oct4 target gene involved in head and neck squamous cell cancer oncogenicity and radioresistance. Oncotarget. 2015;6(1):144-158.
OncoTargets and Therapy

\section{Publish your work in this journal}

OncoTargets and Therapy is an international, peer-reviewed, open access journal focusing on the pathological basis of all cancers, potential targets for therapy and treatment protocols employed to improve the management of cancer patients. The journal also focuses on the impact of management programs and new therapeutic agents and protocols on

\section{Dovepress}

patient perspectives such as quality of life, adherence and satisfaction The manuscript management system is completely online and includes a very quick and fair peer-review system, which is all easy to use. Visit http://www.dovepress.com/testimonials.php to read real quotes from published authors. 